\title{
On the integral Tate conjecture for finite fields and representation theory
}

\author{
Benjamin Antieau
}

\begin{abstract}
We describe a new source of counterexamples to the so-called integral Hodge and integral Tate conjectures. As in the other known counterexamples to the integral Tate conjecture over finite fields, ours are approximations of the classifying space of some group BG. Unlike the other examples, we find groups of type $A_{n}$, our proof relies heavily on representation theory, and Milnor's operations vanish on the classes we construct.
\end{abstract}

\section{Introduction}

Recall that the original conjecture of Hodge predicted that the cycle class maps

$$
\mathrm{cl}^{i}: \mathrm{CH}^{i}(X) \rightarrow \mathrm{H}^{2 i}(X, \mathbb{Z})
$$

would be surjective onto the subgroup of $(i, i)$-classes when $X$ is a smooth projective variety. In [AH62] Atiyah and Hirzebruch constructed for each prime $\ell$ a smooth projective complex algebraic variety $X$ and an $\ell$-torsion integral cohomology class $x \in \mathrm{H}^{4}(X, \mathbb{Z})$, necessarily of type $(2,2)$, such that $x$ is not in the image of the cycle class map. They use the differentials $d_{r}$ in the Atiyah-Hirzebruch spectral sequence and argue that $d_{r}$ vanishes on the image of $\mathrm{cl}^{i}$. By studying the cohomology of finite groups, specifically of $(\mathbb{Z} / \ell)^{3}$, they exhibit degree 4 torsion classes not killed by these differentials. Using Künneth, we see that $\mathrm{H}^{*}\left(\mathrm{~B}(\mathbb{Z} / \ell)^{3}, \mathbb{F}_{\ell}\right)$ has three degree 1 generators, $y_{1}, y_{2}, y_{3}$. The class $y=\beta\left(y_{1} y_{2} y_{3}\right) \in \mathrm{H}^{4}\left(\mathrm{~B}(\mathbb{Z} / \ell)^{3}, \mathbb{Z}\right)$ is the class used by Atiyah and Hirzebruch. They show $d_{2 \ell-1}(y) \neq 0$. Any smooth projective variety that looks sufficiently like $\mathrm{B}(\mathbb{Z} / \ell)^{3} \times \mathrm{B} \mathbb{G}_{m}$ then possesses a cohomology class $x \in \mathrm{H}^{4}(X, \mathbb{Z})$ with $d_{2 \ell-1}(x) \neq 0$. Hence, this class cannot be in the image of the cycle class map. The Godeaux-Serre construction is one way to produce such varieties.

Tate's conjecture is for smooth projective varieties $X$ defined over a field $k$ finitely generated over its prime field and for a fixed prime $\ell$ different from $\operatorname{char}(k)$. It asserts that the cycle class maps in $\ell$-adic cohomology

$$
\mathrm{cl}^{i}: \mathrm{CH}^{i}\left(X_{\bar{k}}\right)_{\mathbb{Q}_{\ell}} \rightarrow \bigcup_{U} \mathrm{H}^{2 i}\left(X_{\bar{k}}, \mathbb{Q}_{\ell}(i)\right)^{U} \subseteq \mathrm{H}^{2 i}\left(X_{\bar{k}}, \mathbb{Q}_{\ell}(i)\right)
$$

are surjective, where $U$ varies over all open subgroups of $\operatorname{Gal}(\bar{k} / k)$ and $\bar{k}$ is the algebraic closure of $k$.

Received 19 April 2015, accepted in final form 25 May 2015.

2010 Mathematics Subject Classification 14C15 (primary), 14C30, 55R40 (secondary).

Keywords: Tate conjecture, cycle class maps, classifying spaces of algebraic groups, Chow rings.

This journal is (c) Foundation Compositio Mathematica 2016. This article is distributed with Open Access under the terms of the Creative Commons Attribution Non-Commercial License, which permits non-commercial reuse, distribution, and reproduction in any medium, provided that the original work is properly cited. For commercial re-use, please contact the Foundation Compositio Mathematica.

The author was supported by NSF grant DMS-1461847. 


\section{On the InTEGRAL TATE CONJECTURE}

There is an integral version of Tate's conjecture in which $\mathbb{Q}_{\ell}(i)$ is replaced by $\mathbb{Z}_{\ell}(i)$, but, like the integral Hodge conjecture, it is false and it appears that it was not expected by Tate. Colliot-Thélène and Szamuely adapted the examples of Atiyah and Hirzebruch to this situation in [CTS10, Théorème 2.1]. Non-torsion classes not in the image of the cycle class map (either in singular cohomology over $\mathbb{C}$ or in $\ell$-adic cohomology) were constructed over uncountable fields by Kollár using very general high-degree hypersurfaces in $\mathbb{P}_{\mathbb{C}}^{4}$.

Since the examples mentioned so far use either torsion cohomology classes or an uncountable ground field, Colliot-Thélène and Szamuely asked in [CTS10, Remark 2.2.3] whether a modified integral Tate conjecture asserting the surjectivity of

$$
\mathrm{CH}^{i}\left(X_{\bar{k}}\right)_{\mathbb{Z}_{\ell}} \rightarrow \mathrm{H}^{2 i}\left(X_{\bar{k}}, \mathbb{Z}_{\ell}(i)\right) / \text { torsion }
$$

could still be true for smooth projective varieties over finite fields.

However, in [PY15], Pirutka and Yagita constructed counterexamples to the surjectivity of (1.1) over finite fields for $\ell=2,3,5$. Their examples are smooth projective approximations to the classifying spaces $\mathrm{B}\left(\mathrm{G} \times \mathbb{G}_{m}\right)$ in characteristic $p$ for $\mathrm{G}$ a simply connected affine algebraic group in the following cases: $\mathrm{G}_{2}$ at the prime $\ell=2, \mathrm{~F}_{4}$ at the prime $\ell=3$, and $\mathrm{E}_{8}$ at the prime $\ell=5$. Each of these groups has $\mathrm{H}^{4}(\mathrm{BG}, \mathbb{Z})=\mathbb{Z} \cdot x$ for some generator $x$, and they show that $x$ is not in the image of the cycle class map. This result gives new counterexamples to the integral Hodge conjecture and, by using integral models and reduction modulo $p$, gives counterexamples to the modulo-torsion integral Tate conjecture over finite fields, answering the question of Colliot-Thélène and Szamuely.

Shortly after the appearance of [PY15], Kameko [Kam15] gave examples for all primes $\ell$ by studying $\mathrm{B}\left(\mathrm{SL}_{\ell} \times \mathrm{SL}_{\ell} / \mu_{\ell}\right)$, where $\mu_{\ell}$ is embedded diagonally.

In all of these examples over finite fields, the argument actually goes by embedding the disruptive group $(\mathbb{Z} / \ell)^{3}$ into $\mathrm{G}$ and then showing that some $x \in \mathrm{H}^{4}(\mathrm{BG}, \mathbb{Z})$ restricts to the class $y \in \mathrm{H}^{4}\left(\mathrm{~B}(\mathbb{Z} / \ell)^{3}, \mathbb{Z}\right)$ found by Atiyah and Hirzebruch. In other words, it shows that $d_{2 \ell-1}(x) \neq 0$ in the Atiyah-Hirzebruch spectral sequence.

The purpose of the present paper is to return to these questions and give a different type of argument, for certain groups of type $A_{n}$, relying on representation theory.

Let $\mathrm{G}$ be a quotient of $\mathrm{SL}_{n}$ by a central subgroup $\mathrm{C} \subseteq \mu_{n}$. Then, $\mathrm{H}^{4}(\mathrm{BG}, \mathbb{Z}) \rightarrow \mathrm{H}^{4}\left(\mathrm{BSL}_{n}, \mathbb{Z}\right)$ is injective, and we can write $\mathrm{H}^{4}(\mathrm{BG}, \mathbb{Z})$ as $\mathbb{Z} \cdot n c_{2}\left(\gamma_{n}\right)$, some multiple of $c_{2}$ of the standard $n$-dimensional representation of $\mathrm{SL}_{n}$. Note, however, that unless $\mathrm{C}$ is trivial $\gamma_{n}$ does not descend to a representation of $\mathrm{G}$.

Theorem 1.1. Let $\mathrm{G}$ be either $\mathrm{SL}_{8} / \mu_{2}$ or $\mathrm{SL}_{9} / \mu_{3}$, viewed as complex affine algebraic groups. Then, $\mathrm{H}^{4}(\mathrm{BG}, \mathbb{Z})=\mathbb{Z} \cdot c_{2}$, while the image of $\mathrm{CH}^{2}(\mathrm{BG}) \rightarrow \mathrm{H}^{4}(\mathrm{BG}, \mathbb{Z})$ is either $2 c_{2} \cdot \mathbb{Z}$ or $3 c_{2} \cdot \mathbb{Z}$, respectively.

Standard arguments then give counterexamples to the mod-torsion Hodge and Tate conjectures in characteristic 0 and the Tate conjecture over $\mathbb{F}_{p}$ for $\ell=2,3$ for all but finitely many primes $p$. In particular, we obtain a new proof of the following theorem of Pirutka-Yagita (for $\ell=2,3,5)$ and Kameko (for all $\ell$ ).

Theorem 1.2 ([PY15, Theorem 1.1], [Kam15, Theorem 1.1]). Let $\ell=2,3$. Then, for all primes $p \neq \ell$ there exists a smooth projective variety $X$ over $\mathbb{F}_{p}$ such that the cycle map

$$
\mathrm{cl}^{2}: \mathrm{CH}^{2}\left(X_{\bar{k}}\right)_{\mathbb{Z}_{\ell}} \rightarrow \bigcup_{U} \mathrm{H}^{4}\left(X_{\bar{k}}, \mathbb{Z}_{\ell}(i)\right)^{U} / \text { torsion }
$$




\section{B. Antieau}

is not surjective.

Our arguments not only differ from those used by Pirutka, Yagita, and Kameko, they must differ. Indeed, we show that in the cases of Theorem 1.1 the differential $d_{2 \ell-1}\left(c_{2}\right)$ equals 0 , in contrast to the work of the previous authors. Moreover, if $\rho\left(c_{2}\right)$ is the reduction modulo $\ell$ of $c_{2} \in$ $\mathrm{H}^{4}(\mathrm{BG}, \mathbb{Z})$ for $G$ as in the theorem, then all of Milnor's operations $\mathrm{Q}_{i}$ vanish on $\rho\left(c_{2}\right)$. However, thanks to the Atiyah-Segal completion theorem, some later differential $d_{r}$ in the spectral sequence does detect the non-algebraicity of $c_{2}$; see Proposition 2.5.

The approach we discovered is checkable for $\ell=2,3$ and in theory for $\mathrm{SL}_{\ell^{2}} / \mu_{\ell}$ for any given odd prime $\ell$. Namely, we use the fact that $\mathrm{CH}^{2}(\mathrm{BG})$ is generated by the Chern classes of polynomial representations of $\mathrm{G}$. We then determine generators for the representation rings of $\mathrm{SL}_{8} / \mu_{2}$ and $\mathrm{SL}_{9} / \mu_{3}$ and compute, using a short SAGE $\left[\mathrm{S}^{+} 13\right]$ program, the second Chern classes of all of these representations. The theorem follows immediately from this computation.

Conjecture 1.3. If $\ell$ is an odd prime, the image of $\mathrm{CH}^{2}(\mathrm{BG}) \rightarrow \mathrm{H}^{4}(\mathrm{BG}, \mathbb{Z})=\mathbb{Z} \cdot c_{2}$ is generated by $\ell c_{2}$ for $\mathrm{G}=\mathrm{SL}_{\ell^{2}} / \mu_{\ell}$.

This paper is organized as follows. In Section 2 we prove Theorem 1.1. In Section 3 we construct smooth projective examples and reduce modulo $p$. Finally, in Section 4 we explain the SAGE code we use for computations.

\section{Examples in characteristic 0}

In this section we work with affine algebraic groups $G$ over the complex numbers. Implicitly, by $\mathrm{H}^{*}(\mathrm{BG}, \mathbb{Z})$ we mean $\mathrm{H}^{*}(\mathrm{BG}(\mathbb{C}), \mathbb{Z})$ and so on. We will use the following result from Totaro's book, which can also be found in the work of Esnault-Kahn-Levine-Viehweg.

Theorem 2.1 ([Tot14, Theorem 2.25], [EKLV98, Lemma C.3]). If $\mathrm{G}$ is an affine algebraic group scheme over a field $k$, then $\mathrm{CH}^{2}(\mathrm{BG})$ is generated by Chern classes of polynomial representation.

Note that the statement in the book is for modulo $\ell$ Chow groups, but that the proof works integrally for $\mathrm{CH}^{2}(\mathrm{BG})$.

We say that the integral Hodge conjecture holds in codimension 2 for $B G$, where $\mathrm{G}$ is an affine algebraic group over $\mathbb{C}$ if $\mathrm{H}^{4}(\mathrm{BG}, \mathbb{Z})$ is generated by Chern classes of representations. This is justified by Theorem 2.1 and the fact that when it fails one can construct counterexamples to the integral Hodge conjecture in codimension 2 on smooth projective complex varieties.

\subsection{Groups of type $A_{n}$ for small $n$}

It is well known that for $\mathrm{G}=\mathrm{SL}_{n}$ and $\mathrm{G}=\mathrm{GL}_{n}$ the cycle class maps give ring isomorphisms $\mathrm{CH}^{*}(\mathrm{BG}) \rightarrow \mathrm{H}^{*}(\mathrm{BG}, \mathbb{Z})$. See [Tot14, Chapter 2]. In other low-dimensional cases, we can obtain a similar conclusion, at least for $\mathrm{H}^{4}(\mathrm{BG}, \mathbb{Z})$. Write $\gamma_{n}$ for the standard $n$-dimensional representation of $\mathrm{SL}_{n}$.

Proposition 2.2. For $\mathrm{G}=\mathrm{PGL}_{2}, \mathrm{PGL}_{3}, \mathrm{SL}_{4} / \mu_{2}, \mathrm{PGL}_{4}, \mathrm{PGL}_{5}, \mathrm{SL}_{6} / \mu_{2}, \mathrm{SL}_{6} / \mu_{3}, \mathrm{PGL}_{6}$, or $\mathrm{PGL}_{7}$, the group $\mathrm{H}^{4}(\mathrm{BG}, \mathbb{Z})$ is generated by Chern classes of polynomial representations.

Proof. For $\mathrm{PGL}_{3}, \mathrm{PGL}_{5}$, and $\mathrm{PGL}_{7}$, this follows from the work of Vistoli [Vis07], and also from Vezzosi [Vez00] for $\mathrm{PGL}_{3}$. Indeed, Vistoli shows more generally that $\mathrm{CH}^{*}\left(\mathrm{BPGL}_{p}\right) \rightarrow$ $\mathrm{H}^{*}\left(\mathrm{BPGL}_{p}, \mathbb{Z}\right)$ is an isomorphism onto the even cohomology for $p$ an odd prime. The case of 


\section{On the InTEGRAL TATE CONJECTURE}

$\mathrm{PGL}_{2} \cong \mathrm{SO}_{3}$ follows from Pandharipande's calculations in [Pan98]. The claim now follows from Theorem 2.1.

The group $\mathrm{H}^{4}\left(\mathrm{BPGL}_{n}, \mathbb{Z}\right) \subseteq \mathrm{H}^{4}\left(\mathrm{BSL}_{n}, \mathbb{Z}\right)=\mathbb{Z} \cdot c_{2}$ is generated by $2 n c_{2}$ for $n$ even and $n c_{2}$ for $n$ odd. On the other hand, the adjoint representation has $c_{2}\left(\mathrm{Ad}_{\mathrm{PGL}_{n}}\right)=2 n c_{2}$. For details, see [Woo82, Sections 1, 2]. For $n$ even it then follows that $\mathrm{H}^{4}\left(\mathrm{BPGL}_{n}, \mathbb{Z}\right)$ is generated by Chern classes, so this takes care of $\mathrm{PGL}_{4}$ and $\mathrm{PGL}_{6}$.

One checks, as we will do in detail for $\mathrm{SL}_{8} / \mu_{2}$ in the next section, that in the remaining cases we have

$$
\begin{aligned}
& \mathrm{H}^{4}\left(\mathrm{BSL}_{4} / \mu_{2}, \mathbb{Z}\right)=\mathbb{Z} \cdot 2 c_{2}, \\
& \mathrm{H}^{4}\left(\mathrm{BSL}_{6} / \mu_{2}, \mathbb{Z}\right)=\mathbb{Z} \cdot 4 c_{2}, \\
& \mathrm{H}^{4}\left(\mathrm{BSL}_{6} / \mu_{3}, \mathbb{Z}\right)=\mathbb{Z} \cdot 3 c_{2} .
\end{aligned}
$$

In these cases, $c_{2}\left(\wedge^{2} \gamma_{4}\right)=2 c_{2}, c_{2}\left(\wedge^{2} \gamma_{6}\right)=4 c_{2}, c_{2}\left(\wedge^{3} \gamma_{6}\right)=6 c_{2}$, and $c_{2}\left(\gamma_{6}^{(2,1)}\right)=33 c_{2}$, where $\gamma_{6}^{(2,1)}$ is the irreducible representation of $\mathrm{SL}_{6}$ corresponding to the partition $(2,1)$. See Section 4 for details. In particular, we see that Chern classes generate $\mathrm{H}^{4}(\mathrm{BG}, \mathbb{Z})$ in each of the remaining cases. These representations all descend to the quotient groups because they are trivial on the given central subgroups.

\subsection{The group $\mathrm{SL}_{8} / \mu_{2}$}

Let $G=\mathrm{SL}_{8} / \mu_{2}$ over $\mathbb{C}$.

TheOrEm 2.3. The pullback map $\mathrm{H}^{4}(\mathrm{BG}, \mathbb{Z}) \rightarrow \mathrm{H}^{4}\left(\mathrm{BSL}_{8}, \mathbb{Z}\right)=\mathbb{Z} \cdot c_{2}$ is an isomorphism, while the image of the cycle class map $\mathrm{cl}^{2}$ is $\mathbb{Z} \cdot 2 c_{2}$ in $\mathrm{H}^{4}(\mathrm{BG}, \mathbb{Z})$. In particular, the integral Hodge conjecture fails in codimension 2 for $\mathrm{BG}=\mathrm{BSL}_{8} / \mu_{2}$.

Proof. Consider the $\mathrm{E}_{2}$-page of the Leray-Serre spectral sequence for the fibration $\mathrm{B} \mu_{2} \rightarrow$ $\mathrm{BSL}_{8} \rightarrow$ BG displayed in Figure 1.

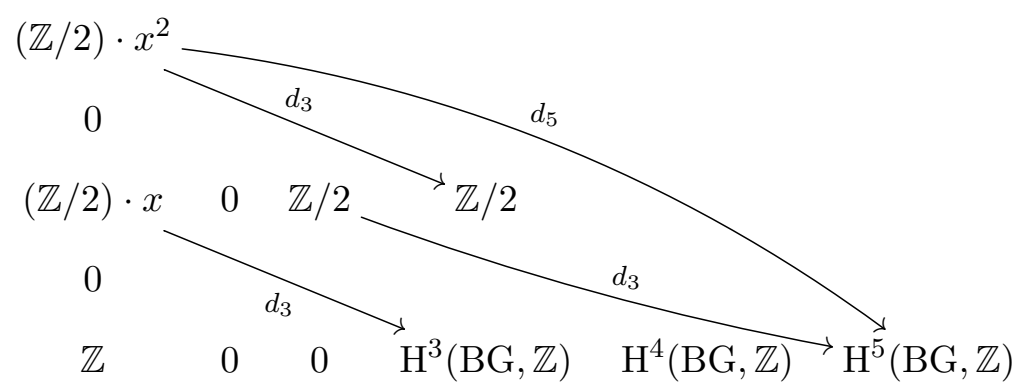

Figure 1. The $\mathrm{E}_{2}$-page of the Leray-Serre spectral sequence associated with $\mathrm{B} \mu_{2} \rightarrow \mathrm{BSL}_{8} \rightarrow$ $\mathrm{BSL}_{8} / \mu_{2}$.

The differential $d_{3}^{0,2}$ is an isomorphism as $\mathrm{H}^{3}(\mathrm{BG}, \mathbb{Z}) \cong \mathbb{Z} / 2$, and we know that $\mathrm{H}^{*}\left(\mathrm{BSL}_{8}, \mathbb{Z}\right)$ is torsion-free. Using the Leibniz rule, we see that $d_{3}\left(x^{2}\right)=0$. Moreover, $\mathrm{H}^{5}(\mathrm{BG}, \mathbb{Z}) \cong \mathbb{Z} / 4$ by the proof of [AW14, Proposition 6.2]. It follows that $d_{3}^{2,2}$ is non-zero, as is $d_{5}^{4,0}$, for otherwise there would be torsion in $\mathrm{H}^{5}\left(\mathrm{BSL}_{8}, \mathbb{Z}\right)$. This shows that the edge map $\mathrm{H}^{4}(\mathrm{BG}, \mathbb{Z}) \rightarrow \mathrm{H}^{4}\left(\mathrm{BSL}_{8}, \mathbb{Z}\right)$ is an isomorphism. 


\section{B. Antieau}

It follows that given a cycle $x \in \mathrm{CH}^{2}(\mathrm{BG})$, we can write $\mathrm{cl}^{2}(x)=n_{x} c_{2}$, where $c_{2} \in \mathrm{H}^{4}\left(\mathrm{BSL}_{8}, \mathbb{Z}\right)$ is the Chern class of the standard representation $\gamma_{8}$ of $\mathrm{SL}_{8}$. We will see that $n_{x}$ is always even.

By Theorem 2.1, to finish the proof it is enough to compute the second Chern classes of generating representations of the representation $\operatorname{ring} \mathrm{R}[\mathrm{G}]$. Let $L_{1}, \ldots, L_{8}$ generate the weight lattice of $\mathrm{SL}_{8}$, with fundamental weights $\alpha_{i}=L_{1}+\cdots+L_{i}$ for $1 \leqslant i \leqslant 7$. An irreducible representation of $\mathrm{SL}_{8}$ with highest weight vector $\lambda_{1} L_{1}+\cdots+\lambda_{8} L_{8}$ descends to a representation of $\mathrm{G}$ if and only if $\sum_{i} \lambda_{i}$ is even. Table 1 gives the generators of the weight lattice of $\mathrm{G}$, the associated Young diagrams, and the numbers $n_{\lambda}$ such that $c_{2}\left(\gamma_{8}^{\lambda}\right)=n_{\lambda} c_{2}\left(\gamma_{8}\right)=n_{\lambda} c_{2}$ in $\mathrm{H}^{4}\left(\mathrm{BSL}_{8}, \mathbb{Z}\right)$. See Section 4 for how the table was computed. By inspection, these Chern classes are all even with greatest common divisor 2, from which the theorem follows.

\begin{tabular}{|c|c|c||c|c|c|}
\hline Weight & $\lambda$ & $n_{\lambda}$ & Weight & $\lambda$ & $n_{\lambda}$ \\
\hline $2 \alpha_{1}$ & $(2)$ & 16 & $\alpha_{1}+\alpha_{3}$ & $(2,1,1)$ & 156 \\
$\alpha_{2}$ & $(1,1)$ & 6 & $\alpha_{1}+\alpha_{5}$ & $(2,1,1,1,1)$ & 170 \\
$2 \alpha_{3}$ & $(2,2,2)$ & 700 & $\alpha_{1}+\alpha_{7}$ & $(2,1,1,1,1,1,1)$ & 16 \\
$\alpha_{4}$ & $(1,1,1,1)$ & 20 & $\alpha_{3}+\alpha_{5}$ & $(2,2,2,1,1)$ & 1344 \\
$2 \alpha_{5}$ & $(2,2,2,2,2)$ & 700 & $\alpha_{3}+\alpha_{7}$ & $(2,2,2,1,1,1,1)$ & 170 \\
$\alpha_{6}$ & $(1,1,1,1,1,1)$ & 6 & $\alpha_{5}+\alpha_{7}$ & $(2,2,2,2,2,1,1)$ & 156 \\
$2 \alpha_{7}$ & $(2,2,2,2,2,2,2)$ & 10 & & & \\
\hline
\end{tabular}

TABLE 1. The second Chern classes of generators of $\mathrm{R}\left[\mathrm{SL}_{8} / \mu_{2}\right]$

The class $c_{2} \in \mathrm{H}^{4}(\mathrm{BG}, \mathbb{Z})$ is a non-torsion counterexample to the integral Hodge conjecture, which we see directly from representation theory. However, it is nevertheless important to know that some cohomology operation tells us that $c_{2}$ cannot be a cycle class. This is critical when we want to pass from BG to a smooth projective variety, either over $\mathbb{C}$ or over a finite field.

In all previous group-theoretic counterexamples at the prime $\ell=2$, such as those of AtiyahHirzebruch, Totaro, Pirutka-Yagita, or Kameko, one has $d_{3}(x)=\mathrm{Sq}_{\mathbb{Z}}^{3}(x) \neq 0$, where $d_{3}=\mathrm{Sq}^{3}$ is the differential in the Atiyah-Hirzebruch spectral sequence converging to $\mathrm{KU}^{*}(\mathrm{BG})$, where $\mathrm{KU}$ is complex topological $K$-theory. This is proved by showing more strongly that the Milnor operation $\mathrm{Q}_{1}$ does not vanish on $\rho(x)$. In our case, we have $d_{3}\left(c_{2}\right)=0$, and moreover $\mathrm{Q}_{i}\left(\rho\left(c_{2}\right)\right)=0$ for all Milnor operations $\mathrm{Q}_{i}$, where $\rho: \mathrm{H}^{*}(\mathrm{BG}, \mathbb{Z}) \rightarrow \mathrm{H}^{*}(\mathrm{BG}, \mathbb{Z} / 2)$.

Recall that the Milnor operations are stable cohomology operations

$$
\mathrm{Q}_{i}: \mathrm{H}^{m}(X, \mathbb{Z} / \ell) \rightarrow \mathrm{H}^{m+2 \ell^{i}-1}(X, \mathbb{Z} / \ell) .
$$

These were defined in motivic cohomology as well by Voevodsky [Voe12, Section 9], to which we refer the reader for an overview. The key point is that $\mathrm{Q}_{i}$ vanishes on the image of the cycle class map.

Theorem 2.4. Let $c_{2} \in \mathrm{H}^{4}(\mathrm{BG}, \mathbb{Z})$ be the generator, where $\mathrm{G}=\mathrm{SL}_{8} / \mu_{2}$, and let $x=\rho\left(c_{2}\right)$. Then, $\mathrm{Q}_{i}(x)=0$ for all $i \geqslant 0$.

Proof. The operation $\mathrm{Q}_{0}$ is the Bockstein operation, so it follows immediately that $\mathrm{Q}_{0}(x)=0$ since $x$ is the reduction of an integral class. The argument that $\mathrm{Q}_{1}(x)=0$ is more subtle, as it 


\section{On the Integral TATE CONJECTURE}

involves the Postnikov tower for BG. Consider the commutative diagram of Postnikov sections

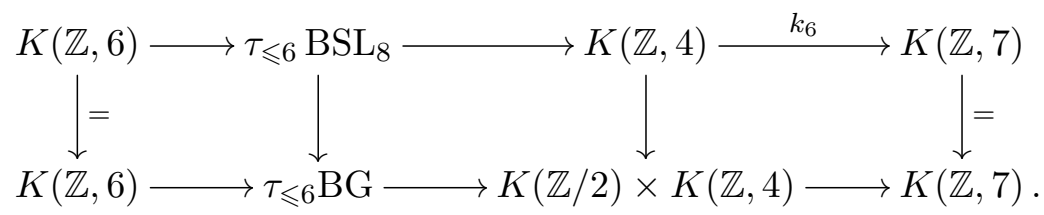

Recall that for a connected topological space $X$ and $n \geqslant 0$, the Postnikov section $\tau_{\leqslant n} X$ is a space with $\pi_{i} \tau_{\leqslant n} X=0$ for $i>n$ equipped with an $n+1$-equivalence $X \rightarrow \tau_{\leqslant n} X$. Since $\mathrm{H}^{7}(K(\mathbb{Z}, 4), \mathbb{Z})=$ $\mathbb{Z} / 2$, and because the cohomology of $\mathrm{BSL}_{8}$ is non-torsion, we see that $k_{6}$ must exactly be the nonzero cohomology class. But, this class is exactly the integral operation $\mathrm{Sq}_{\mathbb{Z}}^{3}$ of the fundamental class of $K(\mathbb{Z}, 4)$. See [AH62, Section 7] for the relationship between the operation $\mathrm{Sq}_{\mathbb{Z}}^{3}$ and $d_{3}$. It follows from the commutative diagram that $d_{3}\left(c_{2}\right)=\mathrm{Sq}_{\mathbb{Z}}^{3}\left(c_{2}\right)=0$ since we construct $\tau_{\leqslant 6} \mathrm{BG}$ using exactly this class as the $k$-invariant. Now, $\mathrm{Q}_{1}(x)=\mathrm{Q}_{1}\left(\rho\left(c_{2}\right)\right)=\rho\left(\mathrm{Sq}_{\mathbb{Z}}^{3}\left(c_{2}\right)\right)=0$.

In general, one has $\mathrm{Q}_{i}=\left[\mathrm{P}^{\ell^{i-1}}, \mathrm{Q}_{i-1}\right]$, the commutator in the Steenrod algebra. The cohomology operations $\mathrm{P}^{i}$ vanish on $\mathrm{H}^{4}(X, \mathbb{Z} / 2)$ for $i>2$ for degree reasons for any space $X$. It follows that it is enough to show $\mathrm{Q}_{2}=0$ because after that all higher Milnor operations will vanish for degree reasons on $\mathrm{P}^{i}$ and by the commutator relation. Since $\mathrm{Q}_{2}=\left[\mathrm{P}^{1}, \mathrm{Q}_{1}\right]=\left[\mathrm{Sq}^{2}, \mathrm{Q}_{1}\right]$ at the prime 2, we have $\mathrm{Q}_{2}(x)=\mathrm{Q}_{1}\left(\mathrm{Sq}^{2}(x)\right)$. Now, $\mathrm{Sq}^{2}(x)=x^{2}$ since $x$ has degree 4 , and we can use that $\mathrm{Q}_{1}=\left[\mathrm{P}^{1}, \mathrm{Q}_{0}\right]$ to see that

$$
\mathrm{Q}_{2}(x)=\mathrm{Q}_{1}\left(\mathrm{Sq}^{2}(x)\right)=\mathrm{Q}_{1}\left(x^{2}\right)=\mathrm{Q}_{0}\left(\mathrm{P}^{1}\left(x^{2}\right)\right)=\mathrm{Q}_{0}\left(\mathrm{Sq}^{1}(x) \mathrm{Sq}^{1}(x)\right)
$$

by the Cartan formula and the fact that $\mathrm{Q}_{0}\left(x^{2}\right)=0$ since $x$ is the reduction of an integral class. For the same reason, $\operatorname{Sq}^{1}(x)=0$, and hence $\mathrm{P}^{2}(x)=0$.

We need to know that nevertheless some cohomology operation detects the non-algebraicity of $c_{2}$. This is provided by the next proposition.

Proposition 2.5. For some odd integer $r>3$, the differential $d_{r}\left(c_{2}\right)$ is non-zero in the AtiyahHirzebruch sequence for $\mathrm{BG}=\mathrm{BSL}_{8} / \mu_{2}$.

Proof. We use the Atiyah-Segal completion theorem [AS69], which says that $\mathrm{KU}^{*}(\mathrm{BG})$ is isomorphic to the completion $\mathrm{R}[\mathrm{G}]_{\widehat{I}}$ of $\mathrm{R}[\mathrm{G}]$ at the augmentation ideal, where $\mathrm{KU}$ denotes complex topological $K$-theory. Strictly speaking, the completion theorem is about compact Lie groups, but in this case there is no difference in terms of either representation theory or $K$-theory in working with $\mathrm{G}=\mathrm{SL}_{8} / \mu_{2}$ versus $\mathrm{SU}_{8} / \mu_{2}$. Both $\mathrm{R}[\mathrm{G}]_{\widehat{I}}$ and $\mathrm{KU}^{0}(\mathrm{BG})$ are naturally filtered, the former by the powers of the augmentation ideal and the latter as the abutment the AtiyahHirzebruch spectral sequence. The Atiyah-Segal isomorphism is compatible with this filtration, which means in particular that the image of $\mathrm{KU}^{*}(\mathrm{BG}) \rightarrow \mathrm{H}^{4}(\mathrm{BG}, \mathbb{Z})$ is the same as the image of $c_{2}: I^{2} / I^{3}$, since $\mathrm{H}^{2}(\mathrm{BG}, \mathbb{Z})=0$. But, we have already found that this map is not surjective, so that it follows that $c_{2}$ must not be permanent in the Atiyah-Hirzebruch spectral sequence. The proposition follows.

\subsection{The group $\mathrm{SL}_{9} / \mu_{3}$}

We take a similar approach for $\mathrm{G}=\mathrm{SL}_{9} / \mu_{3}$ over $\mathbb{C}$.

TheOREM 2.6. The pullback map $\mathrm{H}^{4}(\mathrm{BG}, \mathbb{Z}) \rightarrow \mathrm{H}^{4}\left(\mathrm{BSL}_{9}, \mathbb{Z}\right)=\mathbb{Z} \cdot c_{2}$ is an isomorphism, while the image of the cycle class map $\mathrm{cl}^{2}$ is $\mathbb{Z} \cdot 3 c_{2}$ in $\mathrm{H}^{4}(\mathrm{BG}, \mathbb{Z})$. In particular, the integral Hodge conjecture fails in codimension 2 for $\mathrm{BG}=\mathrm{BSL}_{9} / \mu_{3}$. 


\section{B. Antieau}

Proposition 2.7. For any odd prime $\ell$, the pullback map $\mathrm{H}^{4}(\mathrm{BG}, \mathbb{Z}) \rightarrow \mathrm{H}^{4}\left(\mathrm{BSL}_{\ell^{2}}, \mathbb{Z}\right)=\mathbb{Z} \cdot c_{2}$ is an isomorphism.

Proof. As above, we consider the $\mathrm{E}_{2}$-page of the Leray-Serre spectral sequence $\mathrm{B} \mu_{\ell} \rightarrow \mathrm{BSL}_{\ell^{2}} \rightarrow$ BG displayed in Figure 2.

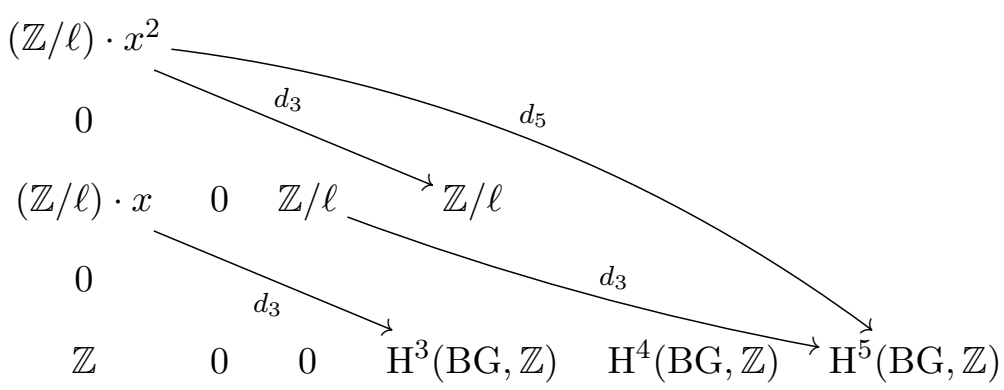

Figure 2. The $\mathrm{E}_{2}$-page of the Leray-Serre spectral sequence associated with $\mathrm{B} \mu_{\ell} \rightarrow \mathrm{BSL}_{\ell^{2}} \rightarrow$ $\mathrm{BSL}_{\ell^{2}} / \mu_{\ell}$.

The main outside input from [AW14, Proposition 6.2] is that $\mathrm{H}^{5}(\mathrm{BG}, \mathbb{Z}) \cong \mathbb{Z} / \ell$. However, in this case, $d_{3}^{0,2}$ is an isomorphism as is $d_{3}^{0,4}$, by the Leibniz rule. It follows that $d_{3}^{2,2}$ is also an isomorphism, from which it follows that the edge map $\mathrm{H}^{4}(\mathrm{BG}, \mathbb{Z}) \rightarrow \mathrm{H}^{4}\left(\mathrm{BSL}_{9}, \mathbb{Z}\right)$ is an isomorphism.

Proof of Theorem 2.6. As above, it suffices to compute the Chern classes of generators of the representation ring of $\mathrm{G}=\mathrm{SL}_{9} / \mu_{3}$. This time there are 23 generators. The results are displayed in Table 2. The theorem follows by inspection.

\begin{tabular}{|c|c|c||c|c|c|}
\hline Weight & $\lambda$ & $n_{\lambda}$ & Weight & $\lambda$ & $n_{\lambda}$ \\
\hline $3 \alpha_{1}$ & $(3)$ & 165 & $2 \alpha_{1}+\alpha_{7}$ & $(3,1,1,1,1,1,1)$ & 693 \\
$3 \alpha_{2}$ & $(3,3)$ & 3465 & $\alpha_{2}+\alpha_{4}$ & $(2,2,1,1)$ & 1701 \\
$\alpha_{3}$ & $(1,1,1)$ & 21 & $\alpha_{2}+\alpha_{7}$ & $(2,2,1,1,1,1,1)$ & 486 \\
$3 \alpha_{4}$ & $(3,3,3,3)$ & 116424 & $2 \alpha_{2}+\alpha_{5}$ & $(3,3,1,1,1)$ & 37125 \\
$3 \alpha_{5}$ & $(3,3,3,3,3)$ & 116424 & $2 \alpha_{2}+\alpha_{8}$ & $(3,3,1,1,1,1,1,1)$ & 2541 \\
$\alpha_{6}$ & $(1,1,1,1,1,1)$ & 21 & $\alpha_{4}+\alpha_{5}$ & $(2,2,2,2,1)$ & 5292 \\
$3 \alpha_{7}$ & $(3,3,3,3,3,3,3)$ & 3465 & $\alpha_{4}+\alpha_{8}$ & $(2,2,2,2,1,1,1,1)$ & 420 \\
$3 \alpha_{8}$ & $(3,3,3,3,3,3,3,3)$ & 66 & $2 \alpha_{4}+\alpha_{7}$ & $(3,3,3,3,1,1,1)$ & 117810 \\
$\alpha_{1}+\alpha_{2}$ & $(2,1)$ & 78 & $\alpha_{5}+\alpha_{7}$ & $(2,2,2,2,2,1,1)$ & 1701 \\
$\alpha_{1}+\alpha_{5}$ & $(2,1,1,1,1)$ & 420 & $2 \alpha_{5}+\alpha_{8}$ & $(3,3,3,3,3,1,1,1)$ & 29106 \\
$\alpha_{1}+\alpha_{8}$ & $(2,1,1,1,1,1,1,1)$ & 18 & $\alpha_{7}+\alpha_{8}$ & $(2,2,2,2,2,2,2,1)$ & 78 \\
$2 \alpha_{1}+\alpha_{4}$ & $(3,1,1,1)$ & 2541 & \multicolumn{3}{|c|}{} \\
\hline \multicolumn{5}{|c|}{ TABLE 2. The second Chern classes of generators of R[SL $\left./ \mu_{3}\right]$} &
\end{tabular}

We include without proof the following results, as the proofs are minor modifications of the proofs of Theorem 2.4 and Proposition 2.5.

Theorem 2.8. Let $c_{2} \in \mathrm{H}^{4}(\mathrm{BG}, \mathbb{Z})$ be the generator, where $\mathrm{G}=\mathrm{SL}_{9} / \mu_{3}$, and let $x=\rho\left(c_{2}\right) \in$ $\mathrm{H}^{4}(\mathrm{BG}, \mathbb{Z} / 3)$. Then, $\mathrm{Q}_{i}(x)=0$ for all $i \geqslant 0$. 


\section{On the InTEGRAL TATE CONJECTURE}

Proposition 2.9. For some odd integer $r>5$, the differential $d_{r}\left(c_{2}\right)$ is non-zero in the cohomology of $\mathrm{BG}=\mathrm{BSL}_{9} / \mu_{3}$.

\subsection{The group $\mathrm{SL}_{\ell^{2}} / \mu_{\ell}$}

It is clear that we cannot simply go on computing representations to get a general result. Nevertheless, for odd primes $\ell>3$ we expect by Proposition 2.7 that a result similar to Theorem 2.6 holds.

Conjecture 2.10. Fix an odd prime $\ell$. Let $\mathrm{G}=\mathrm{SL}_{\ell^{2}} / \mu_{\ell}$ over $\mathbb{C}$. Then, the image of the cycle class map $\mathrm{cl}^{2}$ is the subgroup $\mathbb{Z} \cdot \ell c_{2} \subseteq \mathbb{Z} \cdot c_{2}=\mathrm{H}^{4}(\mathrm{BG}, \mathbb{Z})$.

We intend to return to this question in future work with Ben Williams on the groups $\mathrm{BSL}_{\ell^{2}} / \mu_{\ell}$ and the topological period-index problem.

\section{Counterexamples to the integral Hodge and Tate conjectures}

There is a standard way to pass from counterexamples to the integral Hodge conjecture in the cohomology of BG to actual counterexamples in the cohomology of smooth projective complex varieties. There is also a method for defining these integrally and reducing modulo $p$ to obtain characteristic $p$ counterexamples to the integral Tate conjecture.

We give two modifications to these methods. The first is that we use Poonen's work on Bertini theorems over finite fields [Poo04] to obtain results over every finite field of characteristic different from $\ell$. The second is that we make an argument using Thomason's paper on étale cohomology [Tho85] to show that certain cohomology operations in singular cohomology with

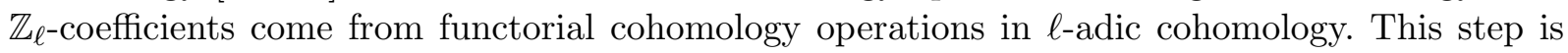
necessary to ensure that upon reducing to finite characteristic we still have access to an operation that detects the non-algebraicity of the class $c_{2}$.

Theorem 3.1. Fix a prime $\ell=2,3$. Let $\mathrm{BG}=\mathrm{BSL}_{8} / \mu_{2}$ or $\mathrm{BSL}_{9} / \mu_{3}$ according to whether $\ell=2$ or $\ell=3$. There exist a smooth projective complex algebraic variety $X$ and a map $f: X \rightarrow \mathrm{BG}$ such that $x=f^{*}\left(c_{2}\right)$ is a non-torsion Hodge class in $\mathrm{H}^{4}(X, \mathbb{Z})$ that is not a cycle class, while $\ell x$ is a cycle class. Moreover, $d_{2 \ell-1}(x)=0$.

Proof. We leave the proof to the reader as an application of Theorems 2.4 and 2.8 and an easy modification of the proof of the next theorem.

The reader should compare the theorem to the original result of Atiyah and Hirzebruch where the non-algebraicity of $x$ is exactly detected by the fact that $d_{2 \ell-1}(x) \neq 0$.

Theorem 3.2 ([PY15, Theorem 1.1], [Kam15, Theorem 1.1]). Let $\ell=2,3$. Then, for all primes $p \neq \ell$ there exists a smooth projective variety $X$ over $\mathbb{F}_{p}$ such that the cycle class map

$$
\mathrm{cl}^{2}: \mathrm{CH}^{2}\left(X_{\bar{k}}\right)_{\mathbb{Z}_{\ell}} \rightarrow \bigcup_{U} \mathrm{H}^{4}\left(X_{\bar{k}}, \mathbb{Z}_{\ell}(i)\right)^{U} / \text { torsion }
$$

is not surjective, where $U$ varies over the open subgroups of $\operatorname{Gal}(\bar{k} / k)$. Moreover, the Milnor operations $\mathrm{Q}_{i}$ vanish on $\mathrm{H}^{4}(X, \mathbb{Z} / \ell(2))$.

Proof. Let $\mathrm{G}=\mathrm{SL}_{8, \mathbb{C}} / \mu_{2}$ or $\mathrm{G}=\mathrm{SL}_{9, \mathbb{C}} / \mu_{3}$, according to the choice of $\ell$. Let $r>3$ be the unique (necessarily odd) integer such that $d_{r}\left(c_{2}\right) \neq 0$ in $\mathrm{H}^{*}(\mathrm{BG}, \mathbb{Z})$, where $d_{r}$ is the differential in the Atiyah-Hirzebruch spectral sequence computing $\mathrm{KU}^{*}(\mathrm{BG})$. 


\section{B. Antieau}

Now, let $\mathrm{G}_{\mathbb{Z}[1 / \ell]}$ denote either $\mathrm{SL}_{8, \mathbb{Z}[1 / 2]} / \mu_{2}$ or $\mathrm{SL}_{9, \mathbb{Z}[1 / 3]} / \mu_{3}$, smooth group-schemes over appropriate localizations of $\mathbb{Z}$. The arguments in [PY15, Section 4] show how to construct a smooth quasi-projective $t$-dimensional scheme $Y \subseteq \mathbb{P}_{\mathbb{Z}[1 / \ell]}^{N}$ over Spec $\mathbb{Z}[1 / \ell]$ such that there is a map $Y \rightarrow \mathrm{B} \mathbb{G}_{m, \mathbb{Z}[1 / \ell]} \times \mathrm{BG}_{\mathbb{Z}[1 \ell]}$ having the following properties:

(i) The induced map $Y_{\mathbb{C}} \rightarrow \mathrm{B} \mathbb{G}_{m, \mathbb{C}} \times \mathrm{BG}_{\mathbb{C}}$ is an $(r+4)$-equivalence.

(ii) The complement $W$ of $Y$ in the closure of $Y$ in $\mathbb{P}_{\mathbb{Z}[1 / \ell]}^{N}$ has large codimension $c$ (to be specified below).

Let $s=\operatorname{Spec} \mathbb{F}_{p}$ be a closed point of $\operatorname{Spec} \mathbb{Z}[1 / \ell]($ so $p \neq \ell$ ), and let $\bar{s}$ be the associated geometric point. Write $Y_{s}$ for the closed fiber of $Y$ over $s$.

We claim that there exists a complete intersection $V_{s} \subseteq \mathbb{P}_{\mathbb{F}_{p}}^{N}$ of codimension $t-c+1$ such that $V_{s} \cap Y_{s}$ is a smooth projective variety. It suffices to show that there is a hypersurface $H_{s}$ such that $H_{s} \cap Y_{s}$ is smooth and $\operatorname{dim} H_{s} \cap W_{s}=\operatorname{dim} W_{s}-1$, for then we can simply continue to cut down with hypersurfaces until the boundary is empty. We use Poonen's Bertini theorem with Taylor coefficients [Poo04, Theorem 1.2]. Indeed, if we let $T_{s} \subseteq W_{s}$ be a finite subscheme containing at least one closed point of each irreducible geometric component of $W_{s}$, then Poonen's theorem implies that there is a positive density of hypersurfaces $H_{s}: f=0$ such that $H_{s} \cap Y_{s}$ is smooth of dimension $\operatorname{dim} Y_{s}-1$ and $f$ does not vanish at any point of $T_{s}$. In particular, it follows that $W_{s} \cap H_{s}$ must have dimension (at most) 1 less than the dimension of $W_{s}$, as desired. Iterating this argument, we find $V_{s}$.

Let us prove that $X_{s}=V_{s} \cap Y_{s}$ has the required properties. Since $V_{s}$ is a complete intersection, it lifts to characteristic 0 , that is, to a flat scheme $V$ over the $p$-adics $\mathbb{Z}_{p}$. Moreover, $X=V \cap Y_{\mathbb{Z}_{p}}$ is a smooth projective scheme over the $p$-adic integers. By Hamm's quasi-projective Lefschetz hyperplane theorem [GM88, Section II.1.2],

$$
\mathrm{H}_{\text {ét }}^{i}\left(\mathrm{~B} \mathbb{G}_{m, \mathbb{C}} \times \mathrm{BG}_{\mathbb{C}}, \mathbb{Z}_{\ell}(j)\right) \cong \mathrm{H}_{\text {ét }}^{i}\left(Y_{\mathbb{C}}, \mathbb{Z}_{\ell}(j)\right) \cong \mathrm{H}_{\text {ét }}^{i}\left(X_{\mathbb{C}}, \mathbb{Z}_{\ell}(j)\right)
$$

for $i \leqslant \operatorname{dim}(X)-2=(c-1)-2=c-3$. By choosing $Y$ such that $r+4 \leqslant c-3$ (or $r+7 \leqslant c)$, one finds that the inequality will hold for $i \leqslant r+4$. That it is possible to find such a $Y$ is proven in [Tot99, Remark 1.4].

Using the invariance of étale cohomology for extensions of algebraically closed fields, and proper base change, there are maps

$$
\mathrm{H}_{\text {ét }}^{i}\left(\mathrm{~B} \mathbb{G}_{m, \mathbb{C}} \times \mathrm{BG}_{\mathbb{C}}, \mathbb{Z}_{\ell}(j)\right) \cong \mathrm{H}_{\text {ét }}^{i}\left(Y_{\mathbb{C}}, \mathbb{Z}_{\ell}(j)\right) \cong \mathrm{H}^{i}\left(X_{\mathbb{C}}, \mathbb{Z}_{\ell}(i)\right) \rightarrow \mathrm{H}_{\text {ét }}^{i}\left(X_{\bar{s}}, \mathbb{Z}_{\ell}(j)\right)
$$

which are isomorphisms for $i \leqslant r+4$. Using the comparison isomorphism between $\ell$-adic cohomology and singular cohomology for smooth complex schemes, we see that there is a class $c_{2} \in \mathrm{H}_{\text {ét }}^{4}\left(X_{\bar{s}}, \mathbb{Z}_{\ell}(2)\right)$ corresponding to $c_{2} \in \mathrm{H}^{4}\left(\mathrm{BG}, \mathbb{Z}_{\ell}\right)$. Note that by looking at the Chern classes of representations of $\mathrm{G}$ we see that $\ell c_{2}$ is actually defined (by abuse of notation) in $\mathrm{H}_{\text {ét }}^{4}\left(X, \mathbb{Z}_{\ell}(2)\right)$. Hence, $c_{2}$ defines a $U$-invariant class in $\mathrm{H}_{\text {ét }}^{4}\left(X_{\bar{s}}, \mathbb{Z}_{\ell}(2)\right)$ for some open subgroup $U \subseteq \operatorname{Gal}\left(\overline{\mathbb{F}}_{p}, \mathbb{F}_{p}\right)$.

Now, it suffices to see that $c_{2}$ cannot be in the image of the cycle class map. We do not know of a source for the full $\ell$-adic cohomology operations, which would say something about $d_{r}$ for $r>3$, so we argue as follows. If $U$ is a smooth complex quasi-projective variety, then

$$
\mathrm{H}^{i}\left(U, \mathbb{Z}_{\ell}\right) \cong \mathrm{H}_{\text {ét }}^{i}\left(U, \mathbb{Z}_{\ell}\right),
$$

where the first group is singular cohomology with coefficients in $\mathbb{Z}_{\ell}$, and the second group is $\ell$-adic cohomology, defined as usual by

$$
\mathrm{H}_{\text {ét }}^{i}\left(U, \mathbb{Z}_{\ell}\right)=\lim _{\nu} \mathrm{H}_{\text {ét }}^{i}\left(U, \mathbb{Z} / \ell^{\nu}\right) .
$$




\section{On the InTEGRAL TATE CONJECTURE}

In order to prove this, note that there are functorial comparison isomorphisms $\mathrm{H}^{i}\left(U, \mathbb{Z} / \ell^{\nu}\right) \cong$ $\mathrm{H}_{\text {ét }}^{i}\left(U, \mathbb{Z} / \ell^{\nu}\right)$, where again the first term is singular cohomology with coefficients in $\mathbb{Z} / \ell^{\nu}$. Hence, to prove that (3.1) is an isomorphism, it suffices to show that the natural map

$$
\mathrm{H}^{i}\left(U, \mathbb{Z}_{\ell}\right) \rightarrow \lim _{\nu} \mathrm{H}^{i}\left(U, \mathbb{Z} / \ell^{\nu}\right)
$$

is an isomorphism for any space $U$ having the homotopy type of a finite CW complex. This is possible to prove by hand, but instead we refer to Bousfield and Kan [BK72, Theorem IX.3.1] for the Milnor exact sequence

$$
0 \rightarrow \lim _{\nu}^{1} \mathrm{H}^{i-1}\left(U, \mathbb{Z} / \ell^{\nu}\right) \rightarrow \mathrm{H}^{i}\left(U, \mathbb{Z}_{\ell}\right) \rightarrow \lim _{\nu} \mathrm{H}^{i}\left(U, \mathbb{Z} / \ell^{n} u\right) \rightarrow 0 .
$$

Since $U$ has the homotopy type of a finite CW complex, the cohomology groups $\mathrm{H}^{i-1}\left(U, \mathbb{Z} / \ell^{\nu}\right)$ are finitely generated, so the Mittag-Leffler condition is automatically satisfied, and the $\lim ^{1}$ term vanishes. So, we have proved that (3.1) is an isomorphism for smooth complex quasi-projective varieties $U$.

Let $\mathrm{K}(U)\left[\beta^{-1}\right]_{\ell}$ denote the $\ell$-adic completion of $\mathrm{K} / \ell^{\nu}(U)\left[\beta^{-1}\right]$, the $\ell$-adic Bott-inverted $K$ theory of Thomason [Tho85, Appendix A]. Typically, the homotopy groups of this spectrum are written $\mathrm{K}_{*}(U)\left[\beta^{-1}\right]_{\ell}$. If $U$ is a quasi-projective scheme over $\mathbb{C}$, this is precisely the $\ell$-complete (periodic) complex $K$-theory spectrum of $U$ by [Tho85, Theorem 4.11]: $\mathrm{K}_{m}(U)\left[\beta^{-1}\right]_{\ell} \cong \mathrm{KU}^{-m}(U)_{\ell}$. However, $\mathrm{K}(U)\left[\beta^{-1}\right]$ is defined functorially over any base field, and there is a spectral sequence

$$
\mathrm{E}_{2}^{p, q}=\mathrm{H}_{\text {ét }}^{p}\left(U, \mathbb{Z}_{\ell}\left(\frac{q}{2}\right)\right) \Rightarrow \mathrm{K}_{q-p}(U)\left[\beta^{-1}\right]_{\ell},
$$

which converges in good cases, for instance in the presence of uniform bounds on the $\ell$-adic étale cohomological dimensions of the residue fields of $U$. The $r$ th differential $d_{r}$ has degree $(r, r-1)$. (Implicitly, if $q$ is odd, the $\mathrm{E}_{2}^{p, q}$ entry is 0.) See Thomason [Tho85, Theorem 4.1]. If $U$ is a $\mathbb{C}$-scheme, then the comparison between $\ell$-adic Bott-inverted algebraic $K$-theory and periodic complex $K$-theory is compatible (via the Atiyah-Hirzebruch spectral sequence) with the comparison between singular and étale $\ell$-adic cohomology. In particular, the differential $d_{r}$ leaving the $\mathrm{E}_{r}^{4,4}$-term of the spectral sequence gives a cohomology operation on a subquotient of $\ell$-adic cohomology which is compatible with base change.

In the Atiyah-Hirzebruch spectral sequence for $\mathrm{BG}_{\mathbb{C}}$ we have $d_{r}\left(c_{2}\right) \neq 0$. Using the compatibility established above, we see that $d_{r}\left(c_{2}\right) \neq 0$ in a subquotient of $\mathrm{H}^{r+4}\left(X_{\bar{s}}, \mathbb{Z}_{\ell}(2+(r-1) / 2)\right)$. On the other hand, this operation $d_{r}$ vanishes on the $\ell$-adic cohomology of $\mathrm{BGL}_{n, \overline{\mathbb{F}}_{p}}$, since $r$ must be odd and $\mathrm{BGL}_{n, \overline{\mathbb{F}}_{p}}$ does not support any odd cohomology. As $\mathrm{CH}^{2}\left(X_{\bar{s}}\right)$ is generated by second Chern classes of representations, we see that $c_{2}$ cannot be in the image of the cycle class map.

\section{Appendix: SAGE code}

The following simple SAGE $\left[\mathrm{S}^{+} 13\right]$ script computes the second Chern classes, with help under the hood from Singular [DGPS13] for multivariable polynomials. The function trim serves to truncate polynomials to make the calculation faster by ignoring higher-degree terms. The function c2 returns $n_{p}$, where $c_{2}\left(\gamma_{n}^{p}\right)=n_{p} c_{2}\left(\gamma_{n}\right)$ for a partition $p$. The input $p$ is of the form $\left(p_{1}, \ldots, p_{k}\right)$.

The main work in the code is done by the built-in SAGE function SemiStandardTableaux, which returns all semistandard tableaux on a given partition. As explained in Fulton's book [Ful97], these index a basis of $\gamma^{\lambda}$, which is then used to compute the Chern classes via the 


\section{B. Antieau}

splitting principle. It seems easier to compute in the cohomology of the classifying space of the maximal torus of $\mathrm{GL}_{8}$ or $\mathrm{GL}_{9}$, so the code just cancels out any $c_{1}$ contributions. We do this by subtracting off $k c_{1}^{2}$, where $k$ is the coefficient of $x_{1}^{2}$ in the resulting polynomial. The function then returns the coefficient of $x_{1} x_{2}$.

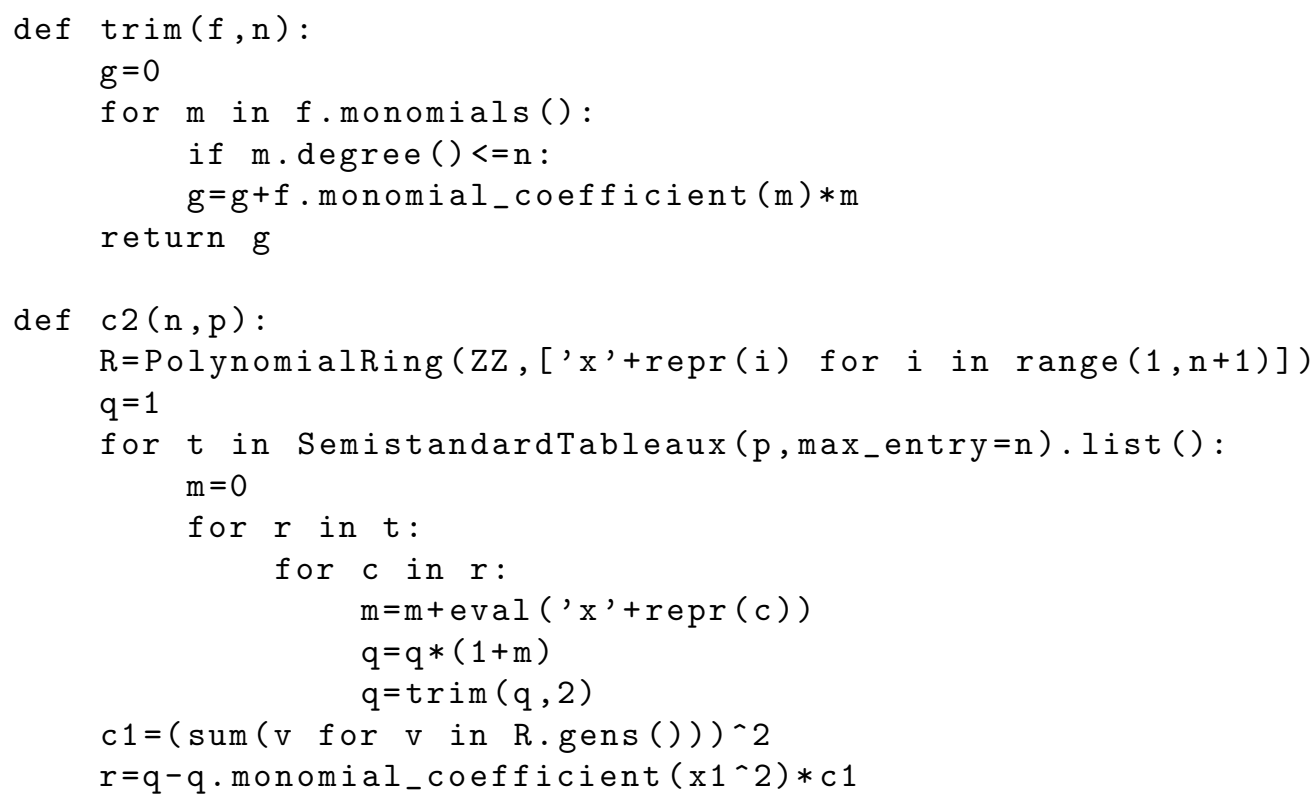

This code will be maintained on the author's github page under the name c2.sage at https://gist.github.com/benjaminantieau and on the author's webpage.

\section{ACKNOWLEDGEMENTS}

We would like to thank Alena Pirutka for sparking our interest in this problem by giving a stimulating talk at the Workshop on Chow groups, motives and derived categories at IAS in March 2015, organized by Totaro and Voisin. We would also like to thank Alena Pirutka, Burt Totaro, and Masaki Kameko for providing us with many useful comments on an early draft of the paper. Special thanks go to Kameko and Totaro for pushing us to prove Theorem 1.2 for all primes $p$.

\section{REFERENCES}

AH62 M.F. Atiyah and F. Hirzebruch, Analytic cycles on complex manifolds, Topology 1 (1962), 25-45; http://dx.doi.org/10.1016/0040-9383(62)90094-0.

AS69 M.F. Atiyah and G. B. Segal, Equivariant K-theory and completion, J. Differential Geom. 3 (1969), 1-18; http://projecteuclid.org/euclid.jdg/1214428815.

AW14 B. Antieau and B. Williams, The topological period-index problem over 6-complexes, J. Topol. 7 (2014), no. 3, 617-640; http://dx.doi.org/10.1112/jtopol/jtt042.

BK72 A. K. Bousfield and D. M. Kan, Homotopy limits, completions and localizations, Lecture Notes in Math., vol. 304 (Springer-Verlag, Berlin - New York, 1972); http://dx.doi.org/10.1007/ 978-3-540-38117-4.

CTS10 J.-L. Colliot-Thélène and T. Szamuely, Autour de la conjecture de Tate à coefficients $\mathbf{Z}_{\ell}$ pour les variétés sur les corps finis, in The Geometry of Algebraic Cycles, Clay Math. Proc., vol. 9 (Amer. Math. Soc., Providence, RI, 2010), 83-98. 


\section{On the Integral TATE CONJeCture}

DGPS13 W. Decker, G.-M. Greuel, G. Pfister, and H. Schönemann, Singular 3-1-5 - A computer algebra system for polynomial computations, 2013, available at http://www.singular.uni-kl.de.

EKLV98 H. Esnault, B. Kahn, M. Levine, and E. Viehweg, The Arason invariant and mod 2 algebraic cycles, J. Amer. Math. Soc. 11 (1998), no. 1, 73-118; http://dx.doi.org/10.1090/ S0894-0347-98-00248-3.

Ful97 W. Fulton, Young tableaux: with applications to representation theory and geometry, London Math. Soc. Stud. Texts, vol. 35, (Cambridge Univ. Press, Cambridge, 1997).

GM88 M. Goresky and R. MacPherson, Stratified Morse theory, Ergeb. Math. Grenzgeb. (3), vol. 14 (Springer-Verlag, Berlin, 1988); http://dx.doi.org/10.1007/978-3-642-71714-7.

Kam15 M. Kameko, On the integral Tate conjecture over finite fields, Math. Proc. Cambridge Philos. Soc. 158 (2015), no. 3, 531-546; http://dx.doi.org/10.1017/S0305004115000134.

Pan98 R. Pandharipande, Equivariant Chow rings of $\mathrm{O}(k), \mathrm{SO}(2 k+1)$, and $\mathrm{SO}(4)$, J. reine angew. Math. 496 (1998), 131-148; http://dx.doi.org/10.1515/crll.1998.025.

Poo04 B. Poonen, Bertini theorems over finite fields, Ann. of Math. (2) 160 (2004), no. 3, 1099-1127; http://dx.doi.org/10.4007/annals.2004.160.1099.

PY15 A. Pirutka and N. Yagita, Note on the counterexamples for the integral Tate conjecture over finite fields, Documenta Math., to appear, arXiv:1401.1620.

$\mathrm{S}^{+} 13$ W. A. Stein et al., Sage Mathematics Software (Version 5.12), The Sage Developers, 2013, available at http://www. sagemath.org.

Tho85 R. W. Thomason, Algebraic K-theory and étale cohomology, Ann. Sci. École Norm. Sup. (4) 18 (1985), no. 3, 437-552; http://www.numdam.org/item?id=ASENS_1985_4_18_3_437_0.

Tot99 B. Totaro, The Chow ring of a classifying space, Algebraic K-Theory (Seattle, WA, 1997), Proc. Sympos. Pure Math., vol. 67 (Amer. Math. Soc., Providence, RI, 1999), 249-281; http: //dx.doi.org/10.1090/pspum/067/1743244.

Tot14 _ Group cohomology and algebraic cycles, Cambridge Tracts in Math., vol. 204, (Cambridge Univ. Press, Cambridge, 2014); http://dx.doi.org/10.1017/CB09781139059480.

Vez00 G. Vezzosi, On the Chow ring of the classifying stack of $\mathrm{PGL}_{3, \mathbf{C}}$, J. reine angew. Math. 523 (2000), 1-54; http://dx.doi.org/10.1515/crll.2000.048.

Vis07 A. Vistoli, On the cohomology and the Chow ring of the classifying space of $\mathrm{PGL}_{p}$, J. reine angew. Math. 610 (2007), 181-227; http://dx.doi.org/10.1515/CRELLE.2007.071.

Voe12 V. Voevodsky, Reduced power operations in motivic cohomology, Publ. Math. Inst. Hautes Études Sci. 98 (2012), no. 1, 1-57; http://dx.doi.org/10.1007/s10240-003-0009-z.

Woo82 L. M. Woodward, The classification of principal $\mathrm{PU}_{n}$-bundles over a 4 -complex, J. London Math. Soc. (2) 25 (1982), no. 3, 513-524; http://dx.doi.org/10.1112/jlms/s2-25.3.513.

Benjamin Antieau benjamin.antieau@gmail.com

Department of Mathematics, Statistics, and Computer Science, University of Illinois at Chicago, 851 S. Morgan St. Chicago, IL 60607, USA 